\title{
Clinical Study to Determine Occult Vestibular Dysfunction in Patients of Chronic Otitis Mediausing Computerized Static Posturography
}

\author{
D K Singh, ${ }^{1}$ Salil Kumar Gupta, ${ }^{1}$ Vijay Bhalla, ${ }^{1}$ Sheetal Raina, ${ }^{1}$ Abha Kumari ${ }^{1}$
}

\begin{abstract}
Introduction:
$\underline{\text { ABSTRACT }}$

Various studies have shown high incidence of subjective vestibular dysfunction in cases of chronic otitis media (COM). Evaluation of vestibular dysfunction in chronic otitis media patients is becoming an integral part of comprehensive management of COM. In our study, we have evaluated vestibular dysfunction in patients with COM, using computerized static posturography, an objective technique in contrast to other subjective tests of vestibular dysfunction.

Materials and Methods

In this prospective case control study done over a period of 1.5 years, 50 cases of COM and 50 healthy controls were included and they underwent Computerized Static Posturography. Parameters taken were Somaesthetic, visual and vestibular scores in both antero-posterior $(A P)$ and medio-lateral (ML) axis; and in combined axis (Antero-posterior + Medio-lateral) and these scores were compared with those of healthy controls.

$\underline{\text { Results }}$

Total 50 cases of COM (average disease period - 5.7 years), of both sexes, with age range of 15-60 years and mean age of 31.58 years were taken. On analyzing above mentioned parameters using SPSS software, we found no significant difference in vestibular function in cases of COM as compared with healthy individuals.

Discussion

The outcome of computerized static posturography can be quantified with respect to changes in center-of-force sway amplitude, distance, or velocity, which, by virtue of not being burdened by subjective interpretation, its results can be, documented both graphically and numerically.

Conclusion

This study, further solidified with objective evidence, raises a question on COM without complications being directly responsible for vestibular dysfunction.

Kevwords:

Otitis Media; Vestibular Dysfunction; Posturography
\end{abstract}

$\mathrm{T}$ The peripheral end organ of the vestibular system is the first sensory system to develop preceding cochlear development and is developed by 49 days of gestation. The vestibular system includes the inner ear (three semicircular canals, the utricle and the

1 - Department of ENT, Command Hospital (Eastern

Command), Kolkata

\section{Corresponding author:}

Dr Salil Kumar Gupta

email: afmcitesalil@gmail.com saccule) and parts of the brain that process the sensory information involved with controlling balance and eye movements. The cochlea and vestibule are anatomically and functionally related to each other and any dysfunction of one or both of them may result in vestibular disorder and subsequent deficit in balance function. ${ }^{1}$

Infection of the inner ear occurs via spread of the pathogenic organism through one of three pathways meninges, middle ear space, or bloodstream. The most commonly reported infection occurs in acute or chronic otitis media (COM) due to passage of infection through 
the round or oval windows. ${ }^{2}$ Both human and animal studies have indicated that the round window is of more significance in the pathologic process. The loss of hair cells occurs due to toxins and enzymes absorbed from the large intercellular spaces in the round window. ${ }^{2,3}$

Various studies done in the past to assess vestibular dysfunction in chronic otitis media patients with or without dizziness or vertigo have shown high incidence of vestibular dysfunction. ${ }^{4}$ Thus diagnosing and evaluating vestibular dysfunction in chronic otitis media patients is essential and should begin with a comprehensive history taking, physical examination and vestibular function tests. ${ }^{4}$

Recently, posturography has become an important part of functional investigation aimed at identifying and assessing vestibular dysfunction. It is an objective technique used to measure postural stability on static or dynamic measuring platforms. Thus it is not burdened by subjective interpretation and the result can be documented both graphically and numerically. Computerized static posturography is carried out by placing the subject in standing pasture on a fixed platform connected to sensitive detectors, which are able to detect the tiny oscillations of the body. ${ }^{5}$

Dynamic posturography involves perturbing the subject's posture by means of a foam cushion or a movable horizontal and tilting platform. As the subject makes small movements, the sensitive detectors transmit this time varying information in real time to a computer. ${ }^{6-8}$ In our study, we have evaluated occult vestibular dysfunction in patients with COM, using computerized static posturography.

\section{Materials and Methods}

After taking approval from the Institutional Ethics Committee, we carried out a prospective case control study in the Department of ENT of this tertiary care hospital over a period of one and half year from October 2013 to March 2015, on patients according to the inclusion criteria, which is as below:

\section{Inclusion Criteria}

1. Patients with unilateral/bilateral COM (either squamous or mucosal) without overt vestibular dysfunction.
2. Patients with age $>5$ years and $<65$ years.

3. Patients without intratemporal or intracranial complications of chronic otitis media.

\section{Exclusion Criteria}

1. Patients who have already undergone previous ear surgery.

2. Patients with acute vertigo or overt vestibular dysfunction.

3. Patients with disorders affecting the visual and proprioceptive functions.

4. Patients with any central cause of vestibular dysfunction.

5. Patients currently on vestibular sedatives.

6. Patients with history of ototoxic drug intake.

Control group included 50 healthy subjects with no ear disease, and no known peripheral/central vestibular dysfunction, and willingness to undergo the procedure.

50 patients with COM were selected consecutively as and when they presented during the study period based on inclusion and exclusion criteria. Written informed consent was taken from the patients, and healthy volunteers.

A pre-designed proforma was used to record relevant information from the individual patients selected, and healthy volunteers. A detailed history was taken from all the patients, and as well the healthy volunteers. Otoscopy and Otomicroscopy (if needed) was carried out to determine the presence of COM and findings recorded, such as type of COM, site etc. All patients underwent Computerized static posturography with Synapsys ${ }^{\circledR}$ posturography System (SPS, SYNAPSIS, Marseille, France) which had inbuilt software for posturography data analysis.

The Synapsys ${ }^{\circledR}$ posturography platform (SPS), allows assessment of static and dynamic balance in the normal,, pathological or aging subject. In addition, it is also possible, with this technique to identify the system(s) responsible for balance dysfunction. During same investigation it can classify the various sensory afferents (visual, vestibular, somaesthetic) involved in postural control, by order of importance; which is what makes it truly unique. The platform, due to its motorized system, is able to produce controlled and reproducible 
Table I: Test conditions during computerized static posturography

\begin{tabular}{|c|c|c|c|}
\hline \multirow{2}{*}{ TEST CONDITION } & PLATFORM & VISION & DURATION \\
\hline 1 & Static & Eyes open & $20 \mathrm{~s}$ \\
\hline 2 & Static & Eyes closed & $20 \mathrm{~s}$ \\
\hline 3 & Unstable surface (foam) & Eyes open & $20 \mathrm{~s}$ \\
\hline 4 & Unstable Surface (foam) & Eyes closed & $20 \mathrm{~s}$ \\
\hline
\end{tabular}

translational movements of the support and study the patients' postural reactions to the provoked disturbances. The software gives pertinent indications to detect postural deficiencies and risk of falls in pathological and/or elderly patients by quantifying objectively their balance performances.

\section{Hardware composition}

The complete system includes:

1. A static platform integrating 3 pressure sensors.

2. A data acquisition system.

3. A software application.

4. A base in foam.

5. An unstable platform.

6. A slip-guard (stepladder + base bars)

7. A translator

The subjects were asked to stand barefoot on the platform with his/her feet aligned to previously determined markings, corresponding to foot size and they were told to stand straight and with arms along the sides of the body, without moving during the whole recording period. Two recordings of each condition were recorded. Evaluation parameters were determined during four test conditions as per Table I.

The parameters evaluated were as follows:

1. Somaesthetic, visual and vestibular scores in both antero-posterior (AP) and medio-lateral (ML) axis.

2. Somaesthetic, visual and vestibular scores in combined axis (Antero-posterior + Medio-lateral).

Data was entered in MS Excel and analyzed using
SPSS 22.0. The Somaesthetic, Visual and Vestibular scores were represented using mean and standard deviation. Comparison of Somaesthetic, Visual and Vestibular scores in normal and COM patients was done using Student's t test.

\section{Results}

Out of fifty COM patients, 30 were males and 20 were females. The age range was from 15-60 years with mean age of 31.58 years. Control group included 50 healthy subjects with no ear disease and no known peripheral/ central vestibular dysfunction, and willingness to undergo the procedure. Out of fifty in this group, 41 were males and 9 were females. The age range was from 20-45 years with mean age of 34.75 years. (Table II)

Tables III, IV, and V compare the mean scores of all three parameters (somaesthetic, visual and vestibular) in antero-posterior (AP), medio-lateral (ML) and combined axis $(\mathrm{AP}+\mathrm{ML})$ between normal and $\mathrm{COM}$ group.

36 patients suffered from mucosal type of COM and 14 suffered from squamous type of COM. The duration of COM was from 1 to 9 years with an average duration of 5.7 years.

On posturography, the mean of the Somaesthetic AP score in normal group was 93.6 with standard deviation of 12.9 while the mean of the Somaesthetic AP score in COM group was 94.0 with standard deviation of 12.7. There was no significant difference in the AP scores of normal and COM groups in Somaesthetic parameter as 
Table II: Demographic data of subjects

\begin{tabular}{|c|c|c|}
\hline VARIABLE & COM PATIENTS & HEALTHY VOLUNTEERS \\
\hline Sample Size & 50 & 50 \\
\hline Male $:$ Female & $3: 2$ & $41: 9$ \\
\hline Age Range & $15-60 \mathrm{yrs}$ & $20-45 \mathrm{yrs}$ \\
\hline Mean Age & $31.58 \mathrm{yrs}$ & $34.75 \mathrm{yrs}$ \\
\hline
\end{tabular}

Table III: Comparison of Somaesthetic, Visual and Vestibular AP SCORES in normal and COM patients

\begin{tabular}{|c|c|c|c|c|c|}
\hline \multirow{2}{*}{ PARAMETERS } & \multicolumn{2}{|c|}{ NORMAL (N=50) } & \multicolumn{2}{c|}{ COM (N=50) } \\
\cline { 2 - 6 } & MEAN & SD & MEAN & SD & P VALUE \\
\cline { 2 - 6 } Somesthetic & 93.6 & 12.9 & 94 & 12.7 & 0.87 \\
\hline Visual & 86.6 & 15.9 & 88.1 & 15.3 & 0.645 \\
\hline Vestibular & 79.6 & 16.6 & 80.4 & 17.2 & 0.823 \\
\hline
\end{tabular}

the p-value was 0.870 .

The mean of the Visual AP score in normal group was 86.6 with standard deviation of 15.9 while the mean of the Visual AP score in COM group was 88.1 with standard deviation of 15.3. The AP scores of normal and COM groups in Visual parameter showed no significant difference as the p-value was 0.645.

The mean of the Vestibular AP score in normal group was 79.6 with standard deviation of 16.6 while the mean of the Vestibular AP score in COM group was 80.4 with standard deviation of 17.2. The AP scores of normal and COM groups in Vestibular parameter also showed no significant difference as the p-value was 0.870 .

The mean of the Somaesthetic ML score in normal group was 96.1 with standard deviation of 10.8 while the mean of the Somaesthetic ML score in COM group was 93.2 with standard deviation of 14.0. There was no significant difference in the ML scores of normal and COM groups in Somaesthetic parameter as the p-value was 0.239 .

The mean of the visual ML score in normal group was 86.7 with standard deviation of 16.2 while the mean of the Visual ML score in COM group was 82.5 with standard deviation of 18.6. The ML scores of normal and COM groups in visual parameter showed no significant difference as the p-value was 0.240 .

The mean of the Vestibular ML score in normal group was 84.9 with standard deviation of 15.6 while the mean of the Vestibular ML score in COM group was 83.1 with standard deviation of 18.4. The AP scores of normal and COM groups in Vestibular parameter also showed no significant difference as the p-value was 0.607 .

The mean of the Somaesthetic and Combined score in normal group was 94.9 with standard deviation of 11.7 
Table IV: Comparison of Somaesthetic, Visual and Vestibular ML SCORES in normal and COM patients

\begin{tabular}{|c|c|c|c|c|c|}
\hline \multirow{2}{*}{ PARAMETERS } & \multicolumn{2}{|c|}{ NORMAL $(\mathbf{N}=\mathbf{5 0})$} & \multicolumn{2}{|c|}{$\operatorname{COM}(\mathrm{N}=50)$} & \multirow{2}{*}{ P VALUE } \\
\hline & MEAN & SD & MEAN & SD & \\
\hline Somesthetic & 96.1 & 10.8 & 93.2 & 14 & 0.239 \\
\hline Visual & 86.7 & 16.2 & 82.5 & 18.6 & 0.24 \\
\hline Vestibular & 84.9 & 15.6 & 83.1 & 18.4 & 0.607 \\
\hline
\end{tabular}

Table V: Comparison of Somaesthetic, Visual and Vestibular COMBINED SCORE in Normal and COM patients

\begin{tabular}{|c|c|c|c|c|c|}
\hline \multirow{2}{*}{ PARAMETERS } & \multicolumn{2}{|c|}{ NORMAL (N=50) } & \multicolumn{2}{c|}{ COM (N=50) } \\
\cline { 2 - 6 } & MEAN & SD & MEAN & SD & \\
\hline Somaesthetic & 94.9 & 11.7 & 93.6 & 12.2 & 0.597 \\
\hline Visual & 86.7 & 13.7 & 85.3 & 14.9 & 0.641 \\
\hline Vestibular & 82.3 & 13.9 & 81.8 & 14 & 0.859 \\
\hline
\end{tabular}

while the mean of the Somaesthetic and Combined score in COM group was 93.6 with standard deviation of 12.2 . There was no significant difference in the combined scores of normal and COM groups in Somaesthetic parameter as the $\mathrm{p}$-value was 0.597 .

The mean of the Visual and Combined score in normal group was 86.7 with standard deviation of 13.7 while the mean of the Visual and combined score in COM group was 85.3 with standard deviation of 14.9. The Combined scores of normal and COM groups in Visual parameter showed no significant difference as the p-value was 0.641 .

The mean of the Vestibular and Combined score in normal group was 82.3 with standard deviation of 13.9 while the mean of the Vestibular and Combined score in COM group was 81.8 with standard deviation of 14.0. The combined scores of normal and COM groups in Vestibular parameter also showed no significant difference as the p-value was 0.859 .

Thus, on comparison of all the three parameters in both the positions (AP \& ML) as well as the combined score, there was no significant difference in patients with COM as compared to normal persons.

\section{Discussion}

This prospective case control study was conducted to detect occult vestibular dysfunction in COM patients using computerized static posturography. Although the objective of this study was to evaluate the vestibular 
aspect of the balance, the results of visual and somaesthetic inputs also have been studied to provide a comprehensive balance evaluation.

Various studies done in the past have shown high incidence of vestibular dysfunction in COM..$^{9-11}$ A study by Lal Siampara et al, in contrast, showed no significant difference in the responses of the diseased and the normal ears on Hot and Cold caloric tests. ${ }^{12}$ They had concluded that majority of the cases of COM have little effect on the vestibular function but long duration of the disease may have an adverse effect.

In our study, no patient with COM was detected to have vestibular dysfunction on the basis of their vestibular scores in anteroposterior and mediolateral axis. Their combined scores also were comparable to healthy controls. Apart from vestibular score, the results of visual and somaesthetic parameters were also evaluated in our study in order to provide a comprehensive balance evaluation. These parameters also did not show statistically significant difference when compared with normal persons.

Thus our study has not demonstrated any significant occult vestibular dysfunction in COM patients as compared to healthy volunteers. Our findings are in line with the study of Lal Siampara et al ${ }^{12}$ but at variance compared to other studies. ${ }^{9-11}$

Vestibular functions in most of the studies done in the past were assessed mainly by caloric testing. The caloric test assesses only the horizontal semicircular canal. Thus, caloric abnormalities do not necessarily imply that the labyrinth is totally dysfunctional and should be interpreted in light of other tests. ${ }^{10}$ In patients of COM open-loop water caloric test cannot be performed due to fear of infection. ${ }^{13}$ Similarly, rotation test (Barany chair), Electronystagmography (ENG) \& Videonystagmography (VNG) have their limitations. ${ }^{6}$, 14,15

It is possible that vestibular dysfunction reported in other studies might be due to limitations of the technique of testing rather than actual vestibular deficit. On the other hand, posturography recently has been accepted as an important part of battery of functional investigations aimed at identifying and assessing vestibular dysfunctions. ${ }^{6}$ It is an objective technique used to measure postural stability on static or dynamic measuring platforms. Thus it is not burdened by subjective interpretation and the results can be documented both graphically and numerically. ${ }^{16}$

Static platform posturography involves stance or tandem stance on a fixed platform with eyes open or closed. This procedure uses the Romberg tests, and the outcome is quantified with respect to changes in centerof-force sway amplitude, distance, or velocity. ${ }^{5}$

Dynamic platform posturography also utilizes Romberg's tests with a fixed platform, but includes test conditions in which the platform and visual environment are moved to reduce the subject's ability to use visual and somatosensory information for balance. All these parameters can also be studied by dynamic posturography. In addition, Dynamic platform posturography incorporates sudden displacements of the platform to test the subject's response to balance perturbations. $^{7,8}$

The reason for not detecting vestibular dysfunction in any COM patient could be due to poor sensitivity of static posturography in identifying occult vestibular dysfunction. Richard P Di Fabio in his study on sensitivity and specificity of platform posturography for detecting vestibular disorders concluded that dynamic and static platform posturography as well as tests of vestibulo-ocular-reflex (VOR) function lack adequate sensitivity to detect vestibular impairment when applied in isolation. ${ }^{16}$ It is possible that addiction of dynamic posturography to our static posturography could have revealed occult vestibular dysfunction in our patients. ${ }^{17,18}$ Most of the studies which found vestibular dysfunction in COM patients, have used both static and dynamic posturography to detect peripheral vestibular dysfunction. ${ }^{19}$

\section{Conclusion}

Hence based on the results of this study we observe that there is no significant difference in vestibular function in cases of COM as compared with healthy individuals when assessed using static posturography. As the sample size of this study is small and has used only static posturography, it is important that a larger 
study be conducted using both static and dynamic posturography to evaluate the presence of occult vestibular dysfunction in COM patients. However, keeping in mind the limitations of static posturography, the findings may differ if dynamic posturography is added to static posturography.

As mentioned above, duration of COM has also been implicated in presence of occult vestibular function. Most of the studies have found vestibular dysfunction in long standing COM cases only, usually more than 10 years. ${ }^{4,19}$ The duration of COM in our patients ranged 1 to 9 years with average of 5.7 years. Hence, it is possible that these patients genuinely did not have vestibular dysfunction and static posturography has detected the same correctly.

\section{Limitations of study -}

1. As per demographic analysis of this study (Table II), although the number of cases in COM group and healthy control group is same, the age and sex variable among two groups are not matched, this decreases the strength of study.

2. The COM group have taken in cluster of patients suffering from both COM Mucosal and Squamous. There is abundance of literature proving that $\mathrm{COM}$ Squamous is more likely to cause complications than COM Mucosal. Although, cases in this study have been clinically examined for any intratemporal or intracranial complications (as per inclusion criteria), but the possibility of a patient suffering from COM Squamous developing occult vestibular complications cannot be negated. However, Labyrinthine fistula, one of the most common complication leading to occult vestibular dysfunction can be caused by both COM Squamous and Mucosal, incidence being 4-13\% among all cases. ${ }^{20,21}$

\section{Financial Support -}

This research received no specific grant from any funding agency, commercial or not-for-profit sectors.

\section{References}

1. Khan S, Chang R. Anatomy of the vestibular system: a review. NeuroRehabilitation 2013; 32(3):437-43
2. Goycoolea MV, Paparella MM, Juhn S, et al. Oval and round window changes in otitis media. Potential pathways between middle and inner ear. Laryngoscope 1980; 90(8):1387-91

3. Cureoglu S, Schachern PA, Rinaldo A, et al. Round window membrane and labyrinthine pathological changes: an overview. Acta Otolaryngol. 2005; 125(1):9-15

4. Mostafa BE, Shafik AG, El Makhzangy AM, et al. Evaluation of vestibular function in patients with chronic suppurative otitis media. ORL 2013; 75(6):357-60

5. GarcíaRB, CorresaSP, BertomeuJMB, etal. Static posturography with dynamic tests. Usefulness of biomechanical parameters in assessing vestibular patients. Acta Otorrinolaringol Esp. 2012; 63(5):332-8

6. Asai M, Watanabe Y, Ohashi N, et al. Evaluation of vestibular function by dynamic posturography and other equilibrium examinations. Acta OtoLaryngol. 1993; 113(sup504):120-4

7. Mirka A, Black FO. Clinical application of dynamic posturography for evaluating sensory integration and vestibular dysfunction. Neurol Clin. 1990; 8(2):351-9

8. Nashner LM, Peters JF. Dynamic Posturography in the Diagnosis and Management of Dizziness and Balance Disorders. Neurol Clin. 1990; 8(2):331-49

9. Schaaf RC. The frequency of vestibular disorders in developmentally delayed preschoolers with otitis media. Am J Occup Ther. 1985; 39(4):247-52

10. Lee I-S, Park HJ, Shin JE, et al. Results of air caloric and other vestibular tests in patients with chronic otitis media. Clin Exp Otorhinolaryngol. 2009; 2(3):145

11. Bhatia R, Deka R. Clinical profile of cases with vertigo. Indian J Otolaryngol Head Neck Surg. 1985; 37(4):144-6

12. Siampara L, Mann S, Panda NK, et al. Audiovestibular profile in unilateral chronic suppurative otitis media. Indian J Otolaryngol Head Neck Surg. 1997; 49(2):107-11

13. Gianoli GJ, Soileau JS. Chronic suppurative otitis media, caloric testing, and rotational chair testing. Otol Neurotol. 2008; 29(1):13-5

14. Schneider E, Glasauer S, Dieterich M. Comparison of human ocular torsion patterns during natural and galvanic vestibular stimulation. J Neurophysiol. 2002; 87(4):2064-73

15. Des Courtis A, Castrillon R, Des Courtis A, et al. Evaluation of subjectivity in the interpretation of videonystagmography. Acta OtoLaryngol. 2008 2008/01/01; 128(8):892-5

16. Di Fabio RP. Sensitivity and specificity of platform posturography for identifying patients with vestibular dysfunction. Phys Ther. 1995; 75(4):290-305

17. Baloh RW, Fife TD, Zwerling L, et al. Comparison of Static and Dynamic Posturography in Young and Older Normal People. J Am Geriatr Soc. 1994; 42(4):405-12

18. Baloh RW, Jacobson KM, Beykirch K, et al. Static and dynamic posturography in patients with vestibular and cerebellar lesions. Arch Neurol. 1998; 55(5):649-54 
19. Monsanto RDC, Kasemodel ALP, Tomaz A, et al. Current evidence of peripheral vestibular symptoms secondary to otitis media. Ann Med. 2018:1-11

20. Browning GG, Weir J, Kelly G, et al. Chronic Otitis Media. In: Watkinson JC, Clarke RW, eds. Scott-Brown's otorhinolaryngology and head and neck surgery. 2. 8 ed. FL: CRC Press, 2018. p. 984

21. Jang CH, Merchant SN. Histopathology of labyrinthine fistulae in chronic otitis media with clinical implications. The American journal of otology. 1997; 18(1):15-25. 